\title{
Measurement of the $\mathbf{Z}+\mathbf{b}$-jet cross-section in pp collisions at $\sqrt{s}=7 \mathrm{TeV}$ in the forward region
}

\section{LHCD}

\section{The LHCb collaboration}

E-mail: murilo.rangel@cern.ch

ABSTRACT: The associated production of a $\mathrm{Z}$ boson or an off-shell photon $\gamma^{*}$ with a bottom quark in the forward region is studied using proton-proton collisions at a centre-of-mass energy of $7 \mathrm{TeV}$. The $\mathrm{Z}$ bosons are reconstructed in the $\mathrm{Z} / \gamma^{*} \rightarrow \mu^{+} \mu^{-}$final state from muons with a transverse momentum larger than $20 \mathrm{GeV}$, while two transverse momentum thresholds are considered for jets $(10 \mathrm{GeV}$ and $20 \mathrm{GeV})$. Both muons and jets are reconstructed in the pseudorapidity range $2.0<\eta<4.5$. The results are based on data corresponding to $1.0 \mathrm{fb}^{-1}$ recorded in 2011 with the $\mathrm{LHCb}$ detector. The measurement of the $\mathrm{Z}+\mathrm{b}$-jet cross-section is normalized to the $\mathrm{Z}+$ jet cross-section. The measured cross-sections are

$$
\sigma\left(\mathrm{Z} / \gamma^{*}\left(\mu^{+} \mu^{-}\right)+\text {b-jet}\right)=295 \pm 60 \text { (stat) } \pm 51 \text { (syst) } \pm 10 \text { (lumi) fb }
$$

for $p_{\mathrm{T}}(\mathrm{jet})>10 \mathrm{GeV}$, and

$$
\sigma\left(\mathrm{Z} / \gamma^{*}\left(\mu^{+} \mu^{-}\right)+\text {b-jet }\right)=128 \pm 36 \text { (stat) } \pm 22 \text { (syst) } \pm 5 \text { (lumi) fb }
$$

for $p_{\mathrm{T}}($ jet $)>20 \mathrm{GeV}$

KEYWORDS: Hadron-Hadron Scattering

ArXIV EPRINT: 1411.1264 


\section{Contents}

1 Introduction 1

2 Detector and samples $\quad 2$

3 Measurement strategy and event selection 2

4 Systematic uncertainties $\quad 4$

5 Results 5

$\begin{array}{lll}6 & \text { Summary } & 7\end{array}$

$\begin{array}{ll}\text { The LHCb collaboration } & 10\end{array}$

\section{Introduction}

The cross-section for the forward production of a $\mathrm{Z}$ boson $^{1}$ in association with a bottom quark (referred to as $\mathrm{Z}+\mathrm{b}-\mathrm{jet}$ ) is sensitive to the parton distribution functions (PDF) in the proton in a phase-space region poorly constrained by existing measurements. It is a benchmark measurement for perturbative quantum chromodynamics phenomenology of heavy quarks and allows constraints to be placed on backgrounds in studies of the Standard Model (SM) Higgs boson and searches for non-SM physics.

The ATLAS and CMS collaborations reported measurements of $\mathrm{Z}+\mathrm{b}$-jet production with jet transverse momentum ${ }^{2}$ larger than $25 \mathrm{GeV}$ and jet pseudorapidity $|\eta|<2.1$, where they find good agreement with next-to-leading order (NLO) predictions [1, 2]. Similar measurements were performed by the CDF [3] and D0 [4] collaborations at the Tevatron, where the dominant contribution comes from the quark-antiquark interaction. The forward acceptance of the LHCb experiment, with a pseudorapidity coverage in the range $2<\eta<5$, probes a kinematic region complementary to that probed by ATLAS and CMS. The LHCb measurements are sensitive to the parton distribution functions in the proton at low and high values of the Bjorken $x$ variable, where the uncertainties are largest.

In this paper we describe the measurement of the production of $\mathrm{Z}+\mathrm{b}$-jet with $\mathrm{Z} / \gamma^{*} \rightarrow$ $\mu^{+} \mu^{-}$in proton-proton collisions at $\sqrt{s}=7 \mathrm{TeV}$ using the data collected by the LHCb experiment in 2011. The data set corresponds to an integrated luminosity of $1.0 \mathrm{fb}^{-1}$.

The presence of a bottom hadron candidate is used to tag the jet as originating from a bottom quark, following ref. [5]. The results are compared to NLO and leading-order (LO) calculations using massless and massive bottom quarks.

\footnotetext{
${ }^{1}$ Throughout this paper $\mathrm{Z}$ boson includes both the $\mathrm{Z}^{0}$ and the off-shell photon, $\gamma^{*}$, contributions.

${ }^{2}$ In this paper we use natural units $(c=\hbar=1)$.
} 


\section{Detector and samples}

The LHCb detector [6] is a single-arm forward spectrometer covering the pseudorapidity range $2<\eta<5$, designed for the study of particles containing $b$ or $c$ quarks. The detector includes a high-precision tracking system consisting of a silicon-strip vertex detector surrounding the $p p$ interaction region [7], a large-area silicon-strip detector located upstream of a dipole magnet with a bending power of about $4 \mathrm{Tm}$, and three stations of silicon-strip detectors and straw drift tubes [8] placed downstream of the magnet. The tracking system provides a measurement of momentum, $p$, with a relative uncertainty that varies from $0.4 \%$ at low momentum to $0.6 \%$ at $100 \mathrm{GeV}$. The minimum distance of a track to a primary vertex, the impact parameter, is measured with a resolution of $\left(15+29 / p_{\mathrm{T}}\right) \mu \mathrm{m}$, where $p_{\mathrm{T}}$ is the transverse momentum in GeV. Different types of charged hadrons are distinguished using information from two ring-imaging Cherenkov detectors [9]. Photon, electron and hadron candidates are identified by a calorimeter system consisting of scintillating-pad (SPD) and preshower detectors, an electromagnetic calorimeter and a hadronic calorimeter. The calorimeters have an energy resolution of $\sigma(E) / E=10 \% / \sqrt{E} \oplus 1 \%$ and $\sigma(E) / E=$ $69 \% / \sqrt{E} \oplus 9 \%$ (with $E$ in $\mathrm{GeV}$ ), respectively. Muons are identified by a system composed of alternating layers of iron and multiwire proportional chambers [10]. The trigger consists of a hardware stage, based on information from the calorimeter and muon systems, followed by a software stage, which applies a full event reconstruction [11].

The events used in this analysis are selected by a trigger that requires the presence of at least one muon candidate with $p_{\mathrm{T}}>10 \mathrm{GeV}$. In addition, the hardware trigger requires a hit multiplicity in the SPD less than 600 , in order to reject events whose processing in the software trigger would be too time consuming. This retains about $90 \%$ of the events that contain a $\mathrm{Z}$ boson.

Simulated samples of $p p$ collisions are generated with PyтHIA v6.4 [12] with a specific LHCb configuration [13] using the CTEQ61l [14] parameterization of the PDFs. Decays of hadronic particles are described by EvTGEN [15], while the interaction of the generated particles with the detector, and its response, are implemented using GEant4 [16] as described in ref. [17].

\section{Measurement strategy and event selection}

The $\mathrm{Z} \rightarrow \mu^{+} \mu^{-}$selection follows that described in ref. [18]. Muon tracks in the fiducial volume $(2.0<\eta(\mu)<4.5)$ are required to have transverse momentum greater than $20 \mathrm{GeV}$. In order to have good quality muons, the relative uncertainty on the momentum of each muon is required to be less than $10 \%$ and the $\chi^{2}$ probability for the associated track fit larger than $0.1 \%$. The dimuon candidate mass is required to be in the $60-120 \mathrm{GeV}$ range. The contribution from combinatorial background of $(0.31 \pm 0.06) \%$, evaluated in ref. [18], is neglected.

Charged and neutral particles are clustered by the anti- $k_{\mathrm{T}}$ algorithm [19] with distance parameter $R=0.5$ as implemented in the FASTJET software package [20]. As in ref. [18], the jet energy is corrected to the particle level excluding neutrinos and the same jet quality requirements are applied. The jets are required to be reconstructed within the pseudora- 

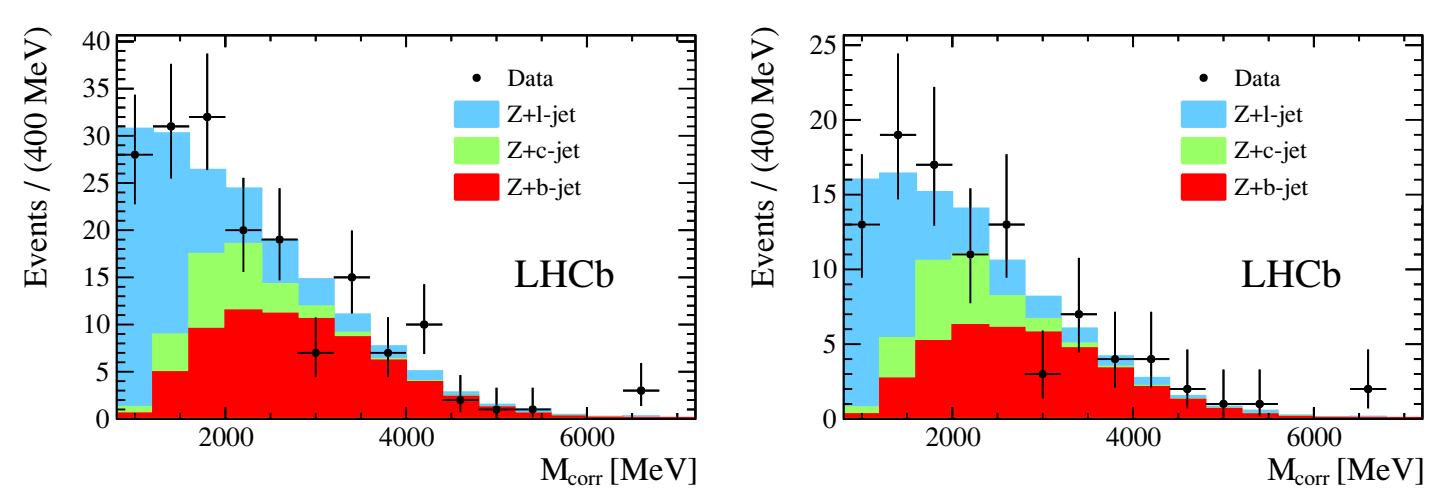

Figure 1. $M_{\text {corr }}$ distribution for (left) $p_{\mathrm{T}}$ (jet) $>10 \mathrm{GeV}$ and (right) $p_{\mathrm{T}}$ (jet) $>20 \mathrm{GeV}$. Data (black points) are compared to the template fit results. The uncertainties shown are statistical only.

pidity range $2.0<\eta$ (jet) $<4.5$ and two transverse momentum thresholds of 10 and $20 \mathrm{GeV}$ are studied. In addition to those kinematic criteria, jets are required to be isolated from the muons of the $\mathrm{Z}$ boson decay $(\Delta \mathrm{R}($ jet,$\mu)>0.4)$, where $\Delta \mathrm{R}$ is the distance in $\eta-\phi$ space and $\phi$ is the azimuthal angle.

The $\mathrm{Z}+\mathrm{b}$-jet cross-section is determined from the ratio of $\mathrm{Z}+\mathrm{b}$-jet to $\mathrm{Z}+$ jet event yields corrected for efficiencies and normalized by the $\mathrm{Z}+$ jet production cross-section

$$
\sigma(\mathrm{Z}+\mathrm{b}-\text { jet })=\frac{\varepsilon(\mathrm{Z}+\text { jet })}{\varepsilon(\mathrm{Z}+\mathrm{b}-\text { jet })} \frac{1}{\varepsilon(\mathrm{b}-\mathrm{tag})} \frac{N(\mathrm{Z}+\mathrm{b}-\mathrm{jet})}{N(\mathrm{Z}+\text { jet })} \sigma(\mathrm{Z}+\text { jet })
$$

where $N(\mathrm{Z}+\mathrm{b}$-jet $)$ is the observed number of $\mathrm{Z}+\mathrm{b}$-jet events, $N(\mathrm{Z}+$ jet $)$ is the number of observed $\mathrm{Z}+$ jet events, $\varepsilon(\mathrm{Z}+$ jet $) / \varepsilon(\mathrm{Z}+\mathrm{b}$-jet $)$ is the ratio of efficiencies for the reconstruction and selection of $\mathrm{Z}+$ jet and $\mathrm{Z}+\mathrm{b}$-jet events and $\varepsilon(\mathrm{b}$-tag) is the efficiency of the b-tagging. The production cross-section of a $\mathrm{Z}$ boson associated with jets, $\sigma(\mathrm{Z}+\mathrm{jet})$, was previously measured by $\mathrm{LHCb}[18]$. The same data sample, $\mathrm{Z}$ boson selection and jet selection are used but identification of jets originating from bottom quarks is added. By using this approach, the systematic uncertainties and the efficiencies are largely the same as those of ref. [18], except for those related to the b-jet identification.

An algorithm similar to that described in refs. [5, 21] is used for the identification of secondary vertices consistent with the decay of a beauty hadron, using tracks that form the jet. Topological secondary vertices (TOPO), significantly separated from the primary vertex, are formed by considering all combinations of two, three and four particles within a jet, where particles include both charged particles reconstruced from tracks and reconstruced $\mathrm{K}_{\mathrm{S}}^{0}$ and $\Lambda$ hadrons. The requirement of a TOPO candidate greatly reduces the background of jets originating from light partons (l-jets) and charm quarks (c-jets). The number of b-jets is extracted from an unbinned likelihood fit to the corrected mass of the TOPO candidate defined as $M_{\text {corr }} \equiv \sqrt{M^{2}+p^{2} \sin ^{2} \theta}+p \sin \theta$. Here, $M$ and $p$ are the invariant mass and momentum of the TOPO candidate and $\theta$ is the angle between its momentum direction and the flight direction inferred from the positions of the primary and secondary vertices [11]. 


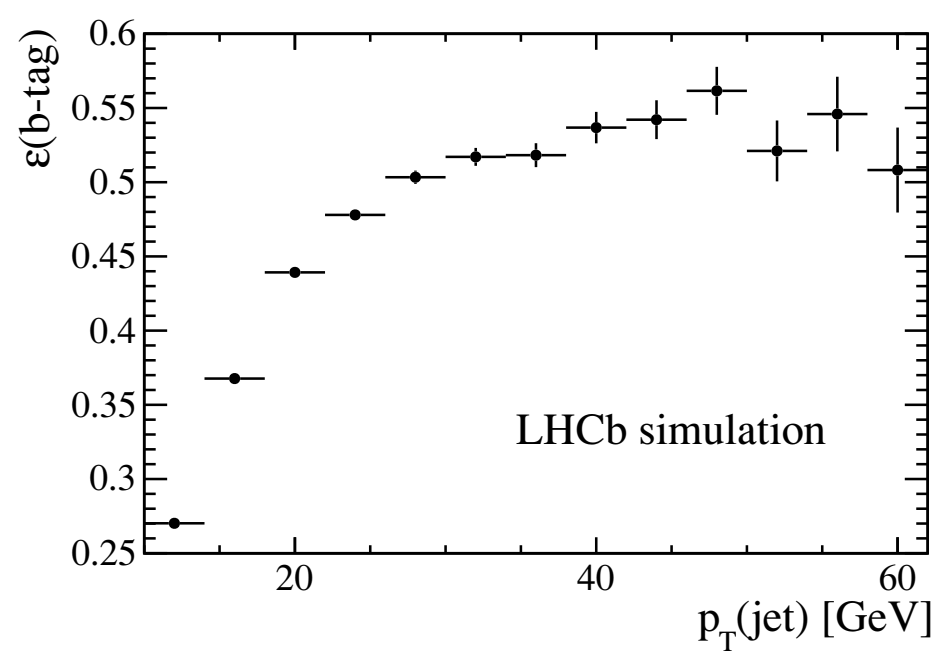

Figure 2. Efficiency of b-tagging as function of the jet transverse momentum. The uncertainties shown are statistical only.

Templates for the $M_{\text {corr }}$ distribution of b-jets, c-jets and l-jets are obtained from simulation of $\mathrm{Z}+$ jet, inclusive b-hadron and inclusive c-hadron production. The shapes of the templates for b-jets, c-jets and l-jets in these samples show no dependence on the production process nor on the $p_{\mathrm{T}}$ of the jet. The $s$ Plot method [22] is used to estimate the b-jet $p_{\mathrm{T}}$ and $\eta$ spectra. Figure 1 shows the $M_{\text {corr }}$ distribution of b-jet candidates with the fit results overlaid.

Jet reconstruction inefficiencies mainly arise from low-momentum particles and calorimeter response, therefore no large differences between jets originating from heavy quarks and from light quarks and gluons are expected. Hence, the ratio $\varepsilon(\mathrm{Z}+$ jet $) / \varepsilon(\mathrm{Z}+\mathrm{b}-\mathrm{jet})$ is assumed to be unity, which is confirmed by simulation.

The b-tagging efficiency, $\varepsilon$ (b-tag), is determined in simulation as a function of the jet transverse momentum and pseudorapidity. The value of $\varepsilon(\mathrm{b}$-tag) shows little variation with pseudorapidity in the range $2.0<\eta($ jet $)<4.5$, while it rises strongly with $p_{\mathrm{T}}$, reaching a value of $55 \%$ at high $p_{\mathrm{T}}$, as shown in figure 2 . The number of $\mathrm{Z}+\mathrm{b}$-jet events determined by the template fit is corrected for the b-tagging efficiency.

\section{Systematic uncertainties}

The systematic uncertainties related to the $\mathrm{Z}$ boson reconstruction, unfolding, jet energy calibration and final-state radiation are taken from ref. [18]. Systematic uncertainties related to the $M_{\text {corr }}$ templates modelling, b-tagging efficiency and jet efficiency flavour dependence are studied in this work.

The systematic uncertainty on the $\mathrm{Z}$ boson reconstruction takes account of the contributions from the track reconstruction, trigger efficiencies, muon identification efficiencies and the model used to fit the $\mathrm{Z}$ boson mass. The $\mathrm{Z}$ boson reconstruction systematic uncertainty is estimated to be $3.5 \%$ [18]. 
Migrations in the jet transverse momentum distribution are corrected for by unfolding. This correction is applied to the value of $\sigma(\mathrm{Z}+$ jet $)$ measured in ref. [18] and used in eq. (3.1). Detailed studies show that no dedicated unfolding correction is necessary. The unfolding systematic uncertainty has two contributions. The difference between the SVD [23] and D'Agostini [24] unfolding methods is assigned as one contribution. The second contribution comes from the difference between the unfolded distribution and the true distribution in an independent simulated sample. This systematic uncertainty is taken from ref. [18] and it is evaluated to be $1.5 \%$.

An important contribution to the systematic uncertainties related to the jets comes from the jet-energy scale. It is estimated by comparing the transverse momentum of the $\mathrm{Z}$ boson and the jet in single jet events, where their momenta are azimuthally opposed, and are expected to be balanced. An additional contribution of $2 \%$ to the jet-energy scale uncertainty comes from the differences between jets initiated from quarks and gluons. The systematic uncertainty of the jet identification is estimated by comparing the number of candidates in data and simulation with a more stringent selection. The total systematic uncertainty related to jets is $7.8 \%$ as estimated in ref. [18].

The systematic uncertainty associated to final-state radiation is obtained by direct comparison to the simulation described above and an additional simulation, using HERWIG $++[25]$, as in ref. [18]; it is estimated to be $0.2 \%$. The systematic uncertainty associated with the knowledge of the luminosity is estimated to be $3.5 \%$ [26].

Possible systematic variations of the final result due to the extraction of $\varepsilon(\mathrm{b}-\mathrm{tag})$ and $M_{\text {corr }}$ templates from simulations are controlled using two data samples enriched in b-jets and c-jets. The b-jet (c-jet) enriched sample is selected via one $B^{ \pm}\left(D^{ \pm}\right)$hadron candidate decaying to $J / \psi K^{ \pm}\left(K^{\mp} \pi^{ \pm} \pi^{ \pm}\right)$produced with a large azimuthal opening angle with respect to a probe jet. The b-tagging requirement is applied to the probe jet and a template fit is performed. Three studies are performed: 1) the data are divided into two ranges of $M$, the template fit is performed on each and the sum of the resulting b-jet yields is compared with the standard result; 2) a looser b-tagging requirement is applied and the b-jet yields after b-tagging efficiency correction are compared with the default values; and 3) the $M_{\text {corr }}$ template is smeared to account for possible differences between simulation and data, and the impact on the b-jet yields is studied. The $M_{\text {corr }}$ simulation modelling and TOPO candidate reconstruction efficiency studies are found to affect this measurement by up to $15 \%$, where this uncertainty is dominated by the first of the studies mentioned above.

Using simulation, $\varepsilon(\mathrm{Z}+$ jet $) / \varepsilon(\mathrm{Z}+\mathrm{b}$-jet $)$ is found to be compatible with unity within $2 \%$, which is taken as the systematic uncertainty due to the flavour dependence of the jet efficiency.

The systematic uncertainties are summarized in table 1 . They are added in quadrature leading to a total systematic error of $17.8 \%$.

\section{Results}

We observe 179 (97) $\mathrm{Z}+$ jet events where at least one jet fulfils the b-tagging requirement for the $p_{\mathrm{T}}($ jet $)>10 \mathrm{GeV}(20 \mathrm{GeV})$ threshold. No events with more than one b-tagged jet 


\begin{tabular}{|lr|}
\hline Source of systematic uncertainty & Relative uncertainty (\%) \\
\hline Z boson reconstruction & 3.5 \\
Unfolding & 1.5 \\
Jet-energy scale, resolution and reconstruction & 7.8 \\
Final-state radiation & 0.2 \\
Luminosity & 3.5 \\
$M_{\text {corr template and b-tagging }}$ & 15.0 \\
Jet reconstruction flavour dependence & 2.0 \\
\hline Total & 17.8 \\
\hline
\end{tabular}

Table 1. Relative systematic uncertainty considered for the $\mathrm{Z}+\mathrm{b}$-jet cross-section for $p_{\mathrm{T}}($ jet $)>$ $20 \mathrm{GeV}$. The relative uncertainties are similar for the $10 \mathrm{GeV}$ threshold. The first four contributions are from ref. [18].

are observed. The extended unbinned likelihood fit of the $M_{\text {corr }}$ spectrum using Z+l-jet, $\mathrm{Z}+\mathrm{c}$-jet and Z+b-jet templates determines $72 \pm 15(39 \pm 11) \mathrm{Z}+\mathrm{b}$-jet events for the $p_{\mathrm{T}}($ jet $)>$ $10 \mathrm{GeV}(20 \mathrm{GeV})$ threshold. The number of candidates corrected for b-tagging efficiency is found to be $177 \pm 36(76 \pm 21)$ for the $p_{\mathrm{T}}($ jet $)>10 \mathrm{GeV}(20 \mathrm{GeV})$ threshold. Using the measurements of ref. [18], we determine the cross-section of $\mathrm{Z}+\mathrm{b}$-jet production to be

$$
\sigma\left(\mathrm{Z} / \gamma^{*}\left(\mu^{+} \mu^{-}\right)+\text {b-jet }\right)=295 \pm 60 \text { (stat) } \pm 51 \text { (syst) } \pm 10 \text { (lumi) fb }
$$

for $p_{\mathrm{T}}($ jet $)>10 \mathrm{GeV}$, and

$$
\sigma\left(\mathrm{Z} / \gamma^{*}\left(\mu^{+} \mu^{-}\right)+\mathrm{b}-\text { jet }\right)=128 \pm 36(\text { stat }) \pm 22(\text { syst }) \pm 5 \text { (lumi) fb }
$$

for $p_{\mathrm{T}}($ jet $)>20 \mathrm{GeV}$. These cross-sections are evaluated within the fiducial region $p_{\mathrm{T}}(\mu)>20 \mathrm{GeV}, 60 \mathrm{GeV}<M\left(\mu^{-} \mu^{+}\right)<120 \mathrm{GeV}, 2.0<\eta($ jet $)<4.5,2.0<\eta(\mu)<4.5$ and $\Delta R($ jet,$\mu)>0.4$.

The measurements are compared to predictions of the $\mathrm{Z}+\mathrm{b}-\mathrm{jet}$ cross-section calculated using MCFM [27] in the same kinematic range as for this measurement. The uncertainties include the PDF and theory uncertainties evaluated by varying independently the renormalization and factorization scales by a factor two around their nominal scales. Neither showering nor hadronization are included in MCFM; therefore the same kinematic requirements applied to jets in the data analysis are applied to the bottom quarks in MCFM. An overall correction is calculated by generating $\mathrm{Z}+\mathrm{b}$-jet events with PYTHIA v8.170 with the MSTW08 PDF set [28] where the same acceptance requirements are applied. Jets are reconstructed with FASTJET using the anti- $k_{\mathrm{T}}$ algorithm with $R=0.5$ and then matched with a bottom quark, requiring $\Delta R$ (jet,b-quark $)<0.5$. The ratio between the number of events with at least one b-jet that fulfils the kinematic requirements of this measurement and the number of events with at least one b quark within the acceptance criteria are used as the fragmentation and hadronization correction for the MCFM predictions. The ratio is 


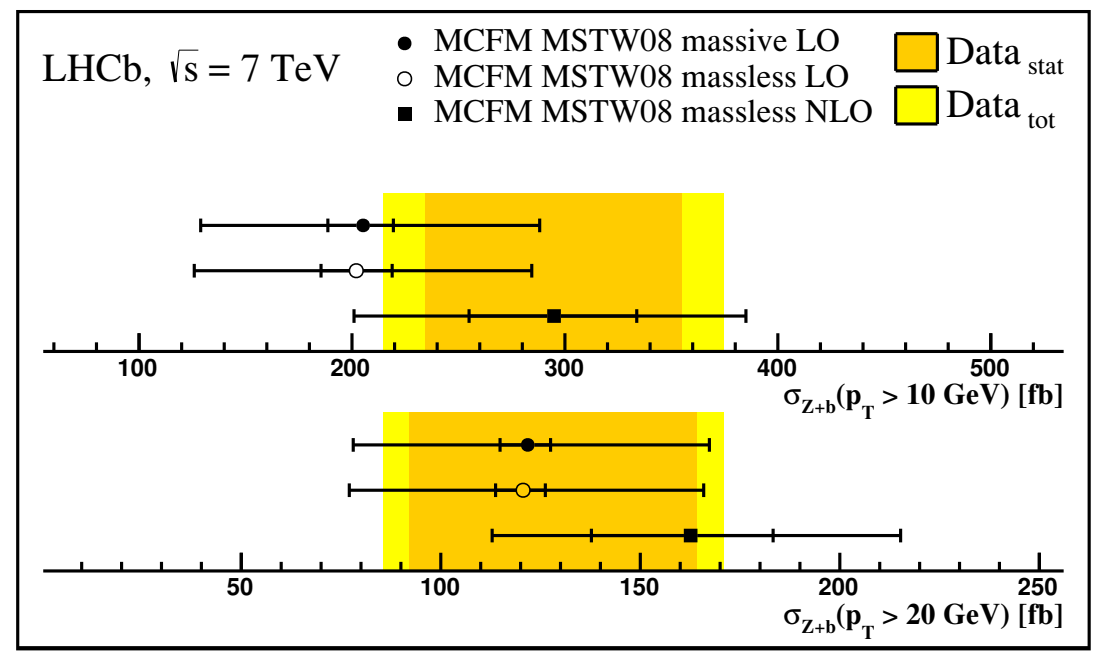

Figure 3. Z+b-jet cross-section for two $p_{\mathrm{T}}$ (jet) thresholds. The colour band shows the LHCb measurement (with the inner orange band showing the statistical uncertainty, and the outer yellow band showing the total uncertainty). The points with error bars correspond to the theoretical predictions with the inner error bars indicating their PDF uncertainties. These cross-sections are evaluated within the fiducial region $p_{\mathrm{T}}(\mu)>20 \mathrm{GeV}, 60 \mathrm{GeV}<M\left(\mu^{-} \mu^{+}\right)<120 \mathrm{GeV}, 2<\eta($ jet $)<$ $4.5,2<\eta(\mu)<4.5$ and $\Delta R($ jet,$\mu)>0.4$.

$0.77(0.90)$ for $p_{\mathrm{T}}($ jet $)>10(20) \mathrm{GeV}$. Figure 3 shows the cross-section measurements compared to the LO calculation with massive bottom quarks and to LO and NLO calculations neglecting the bottom quark mass.

\section{Summary}

The cross-section for forward production of a $\mathrm{Z}$ boson or an off-shell photon, in the $\mu^{+} \mu^{-}$ channel, and a bottom-quark is measured in $\sqrt{s}=7 \mathrm{TeV}$ proton-proton collisions corresponding to an integrated luminosity of $1.0 \mathrm{fb}^{-1}$ of data collected in 2011 by the LHCb collaboration. Results are reported for the kinematic region $2.0<\eta(\mu)<4.5, p_{\mathrm{T}}(\mu)>20 \mathrm{GeV}$, $60<M\left(\mu^{+} \mu^{-}\right)<120 \mathrm{GeV}, p_{\mathrm{T}}($ jet $)>10(20) \mathrm{GeV}, 2.0<\eta($ jet $)<4.5$ and $\Delta \mathrm{R}($ jet, $\mu)>0.4$. The measured cross-sections are

$$
\sigma\left(\mathrm{Z} / \gamma^{*}\left(\mu^{+} \mu^{-}\right)+\mathrm{b} \text {-jet}\right)=295 \pm 60 \text { (stat) } \pm 51 \text { (syst) } \pm 10 \text { (lumi) fb }
$$

for $p_{\mathrm{T}}(\mathrm{jet})>10 \mathrm{GeV}$, and

$$
\sigma\left(\mathrm{Z} / \gamma^{*}\left(\mu^{+} \mu^{-}\right)+\mathrm{b}-\text { jet}\right)=128 \pm 36 \text { (stat) } \pm 22 \text { (syst) } \pm 5 \text { (lumi) fb }
$$

for $p_{\mathrm{T}}($ jet $)>20 \mathrm{GeV}$.

The results are in agreement with MCFM predictions for massless and massive bottom quark calculations. 


\section{Acknowledgments}

We express our gratitude to our colleagues in the CERN accelerator departments for the excellent performance of the LHC. We thank the technical and administrative staff at the LHCb institutes. We acknowledge support from CERN and from the national agencies: CAPES, CNPq, FAPERJ and FINEP (Brazil); NSFC (China); CNRS/IN2P3 (France); BMBF, DFG, HGF and MPG (Germany); SFI (Ireland); INFN (Italy); FOM and NWO (The Netherlands); MNiSW and NCN (Poland); MEN/IFA (Romania); MinES and FANO (Russia); MinECo (Spain); SNSF and SER (Switzerland); NASU (Ukraine); STFC (United Kingdom); NSF (U.S.A.). The Tier1 computing centres are supported by IN2P3 (France), KIT and BMBF (Germany), INFN (Italy), NWO and SURF (The Netherlands), PIC (Spain), GridPP (United Kingdom). We are indebted to the communities behind the multiple open source software packages on which we depend. We are also thankful for the computing resources and the access to software R\&D tools provided by Yandex LLC (Russia). Individual groups or members have received support from EPLANET, Marie SkłodowskaCurie Actions and ERC (European Union), Conseil général de Haute-Savoie, Labex ENIGMASS and OCEVU, Région Auvergne (France), RFBR (Russia), XuntaGal and GENCAT (Spain), Royal Society and Royal Commission for the Exhibition of 1851 (United Kingdom).

Open Access. This article is distributed under the terms of the Creative Commons Attribution License (CC-BY 4.0), which permits any use, distribution and reproduction in any medium, provided the original author(s) and source are credited.

\section{References}

[1] ATLAS collaboration, Measurement of the cross-section for b-jets produced in association with a $Z$ boson at $\sqrt{s}=7 \mathrm{TeV}$ with the ATLAS detector, Phys. Lett. B 706 (2012) 295 [arXiv: 1109.1403] [INSPIRE].

[2] CMS collaboration, Measurement of the $Z / \gamma^{*}+b$-jet cross section in pp collisions at $7 \mathrm{TeV}$, JHEP 06 (2012) 126 [arXiv: 1204.1643] [INSPIRE].

[3] CDF collaboration, T. Aaltonen et al., Measurement of cross sections for $b$ jet production in events with a $Z$ boson in p p collisions at $\sqrt{s}=1.96 \mathrm{TeV}$, Phys. Rev. D 79 (2009) 052008 [arXiv: 0812.4458] [INSPIRE].

[4] D0 collaboration, V.M. Abazov et al., Measurement of the ratio of differential cross sections $\sigma(p \bar{p} \rightarrow Z+b$ jet $) / \sigma(p \bar{p} \rightarrow Z+$ jet $)$ in $p \bar{p}$ collisions at $\sqrt{s}=1.96$ TeV, Phys. Rev. D 87 (2013) 092010 [arXiv:1301.2233] [inSPIRE].

[5] LHCb collaboration, First measurement of the charge asymmetry in beauty-quark pair production, Phys. Rev. Lett. 113 (2014) 082003 [arXiv:1406.4789] [INSPIRE].

[6] LHCb collaboration, The LHCb detector at the LHC, 2008 JINST 3 S08005 [INSPIRE].

[7] R. Aaij et al., Performance of the LHCb vertex locator, 2014 JINST 909007 [arXiv: 1405.7808] [INSPIRE].

[8] LHCb Outer Tracker group collaboration, R. Arink et al., Performance of the LHCb outer tracker, 2014 JINST 9 P01002 [arXiv:1311.3893] [INSPIRE]. 
[9] LHCB RICH Group collaboration, M. Adinolfi et al., Performance of the LHCb RICH detector at the LHC, Eur. Phys. J. C 73 (2013) 2431 [arXiv:1211.6759] [INSPIRE].

[10] A.A. Alves Jr. et al., Performance of the LHCb muon system, 2013 JINST 8 P02022 [arXiv: 1211.1346] [INSPIRE].

[11] R. Aaij et al., The LHCb trigger and its performance in 2011, 2013 JINST 8 P04022 [arXiv: 1211.3055] [INSPIRE].

[12] T. Sjöstrand, S. Mrenna and P.Z. Skands, PYTHIA 6.4 physics and manual, JHEP 05 (2006) 026 [hep-ph/0603175] [INSPIRE].

[13] I. Belyaev et al., Handling of the generation of primary events in Gauss, the LHCb simulation framework, IEEE Nucl. Sci. Symp. Conf. Rec. (NSS/MIC) (2010) 1155.

[14] P.M. Nadolsky et al., Implications of CTEQ global analysis for collider observables, Phys. Rev. D 78 (2008) 013004 [arXiv:0802.0007] [INSPIRE].

[15] D.J. Lange, The EvtGen particle decay simulation package, Nucl. Instrum. Meth. A 462 (2001) 152 [INSPIRE].

[16] GEANT4 collaboration, S. Agostinelli et al., GEANT4: a simulation toolkit, Nucl. Instrum. Meth. A 506 (2003) 250 [INSPIRE].

[17] LHCb collaboration, The LHCb simulation application, Gauss: design, evolution and experience, J. Phys. Conf. Ser. 331 (2011) 032023 [inSPIRE].

[18] LHCb collaboration, Study of forward $Z+$ jet production in pp collisions at $\sqrt{s}=7 \mathrm{TeV}$, JHEP 01 (2014) 033 [arXiv:1310.8197] [INSPIRE].

[19] M. Cacciari, G.P. Salam and G. Soyez, The anti-k $k_{t}$ jet clustering algorithm, JHEP 04 (2008) 063 [arXiv: 0802.1189] [INSPIRE].

[20] M. Cacciari, G.P. Salam and G. Soyez, FastJet user manual, Eur. Phys. J. C 72 (2012) 1896 [arXiv:1111.6097] [INSPIRE].

[21] V.V. Gligorov and M. Williams, Efficient, reliable and fast high-level triggering using a bonsai boosted decision tree, 2013 JINST 8 P02013 [arXiv:1210.6861] [INSPIRE].

[22] M. Pivk and F.R. Le Diberder, SPlot: a statistical tool to unfold data distributions, Nucl. Instrum. Meth. A 555 (2005) 356 [physics/0402083] [INSPIRE].

[23] A. Hocker and V. Kartvelishvili, SVD approach to data unfolding, Nucl. Instrum. Meth. A 372 (1996) 469 [hep-ph/9509307] [INSPIRE].

[24] G. D'Agostini, A multidimensional unfolding method based on Bayes' theorem, Nucl. Instrum. Meth. A 362 (1995) 487 [INSPIRE].

[25] M. Bähr et al., HERWIG++ physics and manual, Eur. Phys. J. C 58 (2008) 639 [arXiv: 0803.0883] [INSPIRE].

[26] LHCb collaboration, Absolute luminosity measurements with the LHCb detector at the LHC, 2012 JINST 7 P01010 [arXiv: 1110.2866] [INSPIRE].

[27] J.M. Campbell and R.K. Ellis, MCFM for the Tevatron and the LHC, Nucl. Phys. Proc. Suppl. 205-206 (2010) 10 [arXiv:1007.3492] [INSPIRE].

[28] A.D. Martin, W.J. Stirling, R.S. Thorne and G. Watt, Parton distributions for the LHC, Eur. Phys. J. C 63 (2009) 189 [arXiv:0901.0002] [InSPIRE]. 


\section{The LHCb collaboration}

R. Aaij ${ }^{41}$, B. Adeva ${ }^{37}$, M. Adinolfi ${ }^{46}$, A. Affolder ${ }^{52}$, Z. Ajaltouni ${ }^{5}$, S. Akar ${ }^{6}$, J. Albrecht $^{9}$, F. Alessio ${ }^{38}$, M. Alexander ${ }^{51}$, S. Ali ${ }^{41}$, G. Alkhazov ${ }^{30}$, P. Alvarez Cartelle ${ }^{37}$, A.A. Alves Jr ${ }^{25,38}$, S. Amato ${ }^{2}$, S. Amerio ${ }^{22}$, Y. Amhis ${ }^{7}$, L. An ${ }^{3}$, L. Anderlini ${ }^{17, g}$, J. Anderson ${ }^{40}$, R. Andreassen ${ }^{57}$, M. Andreotti ${ }^{16, f}$, J.E. Andrews ${ }^{58}$, R.B. Appleby ${ }^{54}$, O. Aquines Gutierrez ${ }^{10}$, F. Archilli ${ }^{38}$, A. Artamonov ${ }^{35}$, M. Artuso ${ }^{59}$, E. Aslanides ${ }^{6}$, G. Auriemma ${ }^{25, n}$, M. Baalouch ${ }^{5}$, S. Bachmann ${ }^{11}$, J.J. Back ${ }^{48}$, A. Badalov ${ }^{36}$, C. Baesso ${ }^{60}$, W. Baldini ${ }^{16}$, R.J. Barlow ${ }^{54}$, C. Barschel ${ }^{38}$, S. Barsuk ${ }^{7}$, W. Barter ${ }^{47}$, V. Batozskaya ${ }^{28}$, V. Battista ${ }^{39}$, A. Bay ${ }^{39}$, L. Beaucourt ${ }^{4}$, J. Beddow ${ }^{51}$, F. Bedeschi ${ }^{23}$, I. Bediaga ${ }^{1}$, S. Belogurov ${ }^{31}$, K. Belous ${ }^{35}$, I. Belyaev ${ }^{31}$, E. Ben-Haim ${ }^{8}$, G. Bencivenni ${ }^{18}$, S. Benson ${ }^{38}$, J. Benton ${ }^{46}$, A. Berezhnoy ${ }^{32}$, R. Bernet ${ }^{40}$, AB Bertolin ${ }^{22}$, M.-O. Bettler ${ }^{47}$, M. van Beuzekom ${ }^{41}$, A. Bien ${ }^{11}$, S. Bifani ${ }^{45}$, T. Bird ${ }^{54}$, A. Bizzeti ${ }^{17, i}$, P.M. Bjørnstad ${ }^{54}$, T. Blake ${ }^{48}$, F. Blanc ${ }^{39}$, J. Blouw ${ }^{10}$, S. Blusk ${ }^{59}$, V. Bocci ${ }^{25}$, A. Bondar ${ }^{34}$, N. Bondar ${ }^{30,38}$, W. Bonivento ${ }^{15}$, S. Borghi ${ }^{54}$, A. Borgia ${ }^{59}$, M. Borsato $^{7}$, T.J.V. Bowcock ${ }^{52}$, E. Bowen ${ }^{40}$, C. Bozzi ${ }^{16}$, D. Brett ${ }^{54}$, M. Britsch ${ }^{10}$, T. Britton ${ }^{59}$, J. Brodzicka ${ }^{54}$, N.H. Brook ${ }^{46}$, A. Bursche ${ }^{40}$, J. Buytaert ${ }^{38}$, S. Cadeddu ${ }^{15}$, R. Calabrese ${ }^{16, f}$, M. Calvi ${ }^{20, k}$, M. Calvo Gomez ${ }^{36, p}$, P. Campana ${ }^{18}$, D. Campora Perez ${ }^{38}$, L. Capriotti ${ }^{54}$, A. Carbone ${ }^{14, d}$, G. Carboni ${ }^{24, l}$,

R. Cardinale ${ }^{19,38, j}$, A. Cardini ${ }^{15}$, L. Carson ${ }^{50}$, K. Carvalho Akiba ${ }^{2,38}$, RCM Casanova Mohr $^{36}$,

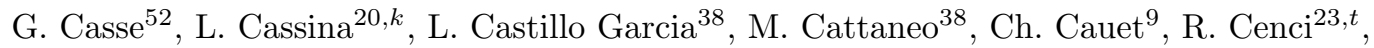

M. Charles ${ }^{8}$, Ph. Charpentier ${ }^{38}$, M. Chefdeville ${ }^{4}$, S. Chen ${ }^{54}$, S.-F. Cheung ${ }^{55}$, N. Chiapolini ${ }^{40}$, M. Chrzaszcz ${ }^{40,26}$, X. Cid Vidal ${ }^{38}$, G. Ciezarek $^{41}$, P.E.L. Clarke ${ }^{50}$, M. Clemencic ${ }^{38}$, H.V. Cliff ${ }^{47}$, J. Closier ${ }^{38}$, V. Coco $^{38}$, J. Cogan ${ }^{6}$, E. Cogneras 5 , V. Cogoni ${ }^{15}$, L. Cojocariu ${ }^{29}$, G. Collazuol ${ }^{22}$, P. Collins ${ }^{38}$, A. Comerma-Montells ${ }^{11}$, A. Contu ${ }^{15,38}$, A. Cook $^{46}$, M. Coombes ${ }^{46}$, S. Coquereau ${ }^{8}$, G. Corti $^{38}$, M. Corvo ${ }^{16, f}$, I. Counts ${ }^{56}$, B. Couturier ${ }^{38}$, G.A. Cowan ${ }^{50}$, D.C. Craik ${ }^{48}$,

A.C. Crocombe ${ }^{48}$, M. Cruz Torres ${ }^{60}$, S. Cunliffe ${ }^{53}$, R. Currie ${ }^{53}$, C. D'Ambrosio ${ }^{38}$, J. Dalseno ${ }^{46}$, P. David ${ }^{8}$, P.N.Y. David ${ }^{41}$, A. Davis ${ }^{57}$, K. De Bruyn ${ }^{41}$, S. De Capua ${ }^{54}$, M. De Cian ${ }^{11}$, J.M. De Miranda ${ }^{1}$, L. De Paula ${ }^{2}$, W. De Silva ${ }^{57}$, P. De Simone ${ }^{18}$, C.-T. Dean ${ }^{51}$, D. Decamp ${ }^{4}$, M. Deckenhoff ${ }^{9}$, L. Del Buono ${ }^{8}$, N. Déléage ${ }^{4}$, D. Derkach $^{55}$, O. Deschamps ${ }^{5}$, F. Dettori ${ }^{38}$, B. Dey $^{40}$, A. Di Canto ${ }^{38}$, A Di Domenico ${ }^{25}$, H. Dijkstra ${ }^{38}$, S. Donleavy ${ }^{52}$, F. Dordei ${ }^{11}$, M. Dorigo ${ }^{39}$, A. Dosil Suárez ${ }^{37}$, D. Dossett ${ }^{48}$, A. Dovbnya ${ }^{43}$, K. Dreimanis ${ }^{52}$, G. Dujany ${ }^{54}$, F. Dupertuis ${ }^{39}$, P. Durante ${ }^{38}$, R. Dzhelyadin ${ }^{35}$, A. Dziurda ${ }^{26}$, A. Dzyuba ${ }^{30}$, S. Easo ${ }^{49,38}$, U. Egede ${ }^{53}$, V. Egorychev ${ }^{31}$, S. Eidelman ${ }^{34}$, S. Eisenhardt ${ }^{50}$, U. Eitschberger ${ }^{9}$, R. Ekelhof ${ }^{9}$, L. Eklund ${ }^{51}$, I. El Rifai ${ }^{5}$, Ch. Elsasser ${ }^{40}$, S. Ely ${ }^{59}$, S. Esen ${ }^{11}$, H.-M. Evans ${ }^{47}$, T. Evans ${ }^{55}$, A. Falabella ${ }^{14}$, C. Färber ${ }^{11}$, C. Farinelli ${ }^{41}$, N. Farley ${ }^{45}$, S. Farry ${ }^{52}$, R. Fay ${ }^{52}$, D. Ferguson ${ }^{50}$, V. Fernandez Albor ${ }^{37}$, F. Ferreira Rodrigues ${ }^{1}$, M. Ferro-Luzzi ${ }^{38}$, S. Filippov ${ }^{33}$, M. Fiore ${ }^{16, f}$, M. Fiorini ${ }^{16, f}$, M. Firlej ${ }^{27}$, C. Fitzpatrick ${ }^{39}$, T. Fiutowski ${ }^{27}$, P. Fol ${ }^{53}$, M. Fontana ${ }^{10}$, F. Fontanelli ${ }^{19, j}$, R. Forty ${ }^{38}$, O. Francisco ${ }^{2}$, M. Frank ${ }^{38}$, C. Frei ${ }^{38}$, M. Frosini ${ }^{17, g}$, J. Fu ${ }^{21,38}$, E. Furfaro ${ }^{24, l}$, A. Gallas Torreira ${ }^{37}$, D. Galli ${ }^{14, d}$, S. Gallorini ${ }^{22,38}$, S. Gambetta ${ }^{19, j}$,

M. Gandelman ${ }^{2}$, P. Gandini ${ }^{59}$, Y. Gao ${ }^{3}$, J. García Pardiñas ${ }^{37}$, J. Garofoli ${ }^{59}$, J. Garra Tico ${ }^{47}$, L. Garrido ${ }^{36}$, D. Gascon ${ }^{36}$, C. Gaspar ${ }^{38}$, U. Gastaldi ${ }^{16}$, R. Gauld ${ }^{55}$, L. Gavardi ${ }^{9}$, G. Gazzoni ${ }^{5}$, A. Geraci ${ }^{21, v}$, E. Gersabeck ${ }^{11}$, M. Gersabeck ${ }^{54}$, T. Gershon ${ }^{48}$, Ph. Ghez $^{4}$, A. Gianelle ${ }^{22}$, S. Gianì ${ }^{39}$, V. Gibson ${ }^{47}$, L. Giubega ${ }^{29}$, V.V. Gligorov ${ }^{38}$, C. Göbel ${ }^{60}$, D. Golubkov ${ }^{31}$, A. Golutvin ${ }^{53,31,38}$, A. Gomes ${ }^{1, a}$, C. Gotti ${ }^{20, k}$, M. Grabalosa Gándara ${ }^{5}$, R. Graciani Diaz ${ }^{36}$, L.A. Granado Cardoso ${ }^{38}$, E. Graugés ${ }^{36}$, E. Graverini ${ }^{40}$, G. Graziani ${ }^{17}$, A. Grecu ${ }^{29}$, E. Greening ${ }^{55}$, S. Gregson ${ }^{47}$, P. Griffith ${ }^{45}$, L. Grillo ${ }^{11}$, O. Grünberg ${ }^{63}$, B. Gui ${ }^{59}$, E. Gushchin ${ }^{33}$, Yu. Guz ${ }^{35,38}$, T. Gys ${ }^{38}$, C. Hadjivasiliou ${ }^{59}$, G. Haefeli ${ }^{39}$, C. Haen ${ }^{38}$, S.C. Haines ${ }^{47}$, S. Hall ${ }^{53}$, B. Hamilton ${ }^{58}$, T. Hampson ${ }^{46}$, X. Han ${ }^{11}$, S. Hansmann-Menzemer ${ }^{11}$, N. Harnew ${ }^{55}$, S.T. Harnew ${ }^{46}$, J. Harrison ${ }^{54}$, J. He ${ }^{38}$, T. Head ${ }^{39}$, V. Heijne ${ }^{41}$, K. Hennessy ${ }^{52}$, P. Henrard ${ }^{5}$, L. Henry ${ }^{8}$, J.A. Hernando Morata ${ }^{37}$, 
E. van Herwijnen ${ }^{38}$, M. Heß ${ }^{63}$, A. Hicheur ${ }^{2}$, D. Hill ${ }^{55}$, M. Hoballah ${ }^{5}$, C. Hombach ${ }^{54}$, W. Hulsbergen ${ }^{41}$, N. Hussain ${ }^{55}$, D. Hutchcroft ${ }^{52}$, D. Hynds ${ }^{51}$, M. Idzik ${ }^{27}$, P. Ilten ${ }^{56}$, R. Jacobsson ${ }^{38}$, A. Jaeger ${ }^{11}$, J. Jalocha ${ }^{55}$, E. Jans ${ }^{41}$, A. Jawahery ${ }^{58}$, F. Jing ${ }^{3}$, M. John ${ }^{55}$, D. Johnson ${ }^{38}$, C.R. Jones ${ }^{47}$, C. Joram ${ }^{38}$, B. Jost ${ }^{38}$, N. Jurik ${ }^{59}$, S. Kandybei ${ }^{43}$, W. Kanso ${ }^{6}$, M. Karacson ${ }^{38}$, T.M. Karbach ${ }^{38}$, S. Karodia ${ }^{51}$, M. Kelsey ${ }^{59}$, I.R. Kenyon ${ }^{45}$, T. Ketel ${ }^{42}$, B. Khanji ${ }^{20,38, k}$, C. Khurewathanakul ${ }^{39}$, S. Klaver ${ }^{54}$, K. Klimaszewski ${ }^{28}$, O. Kochebina ${ }^{7}$, M. Kolpin ${ }^{11}$, I. Komarov ${ }^{39}$, R.F. Koopman ${ }^{42}$, P. Koppenburg ${ }^{41,38}$, M. Korolev ${ }^{32}$, L. Kravchuk ${ }^{33}$, K. Kreplin ${ }^{11}$, M. Kreps ${ }^{48}$, G. Krocker ${ }^{11}$, P. Krokovny ${ }^{34}$, F. Kruse ${ }^{9}$, W. Kucewicz ${ }^{26, o}$, M. Kucharczyk ${ }^{20,26, k}$, V. Kudryavtsev ${ }^{34}$, K. Kurek ${ }^{28}$, T. Kvaratskheliya ${ }^{31}$, V.N. La Thi ${ }^{39}$, D. Lacarrere $^{38}$, G. Lafferty ${ }^{54}$, A. Lai ${ }^{15}$, D. Lambert ${ }^{50}$, R.W. Lambert ${ }^{42}$, G. Lanfranchi ${ }^{18}$, C. Langenbruch ${ }^{48}$, B. Langhans ${ }^{38}$, T. Latham ${ }^{48}$, C. Lazzeroni ${ }^{45}$, R. Le Gac ${ }^{6}$, J. van Leerdam ${ }^{41}$, J.-P. Lees ${ }^{4}$, R. Lefèvre ${ }^{5}$, A. Leflat ${ }^{32}$, J. Lefrançois ${ }^{7}$, O. Leroy ${ }^{6}$, T. Lesiak ${ }^{26}$, B. Leverington ${ }^{11}$,

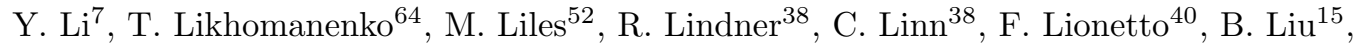
S. Lohn ${ }^{38}$, I. Longstaff ${ }^{51}$, J.H. Lopes ${ }^{2}$, P. Lowdon ${ }^{40}$, D. Lucchesi ${ }^{22, r}$, H. Luo ${ }^{50}$, A. Lupato ${ }^{22}$, E. Luppi ${ }^{16, f}$, O. Lupton ${ }^{55}$, F. Machefert ${ }^{7}$, I.V. Machikhiliyan ${ }^{31}$, F. Maciuc ${ }^{29}$, O. Maev ${ }^{30}$, S. Malde ${ }^{55}$, A. Malinin ${ }^{64}$, G. Manca ${ }^{15, e}$, G. Mancinelli ${ }^{6}$, A. Mapelli $^{38}$, J. Maratas $^{5}$, J.F. Marchand ${ }^{4}$, U. Marconi ${ }^{14}$, C. Marin Benito ${ }^{36}$, P. Marino ${ }^{23, t}$, R. Märki ${ }^{39}$, J. Marks ${ }^{11}$, G. Martellotti ${ }^{25}$, M. Martinelli ${ }^{39}$, D. Martinez Santos ${ }^{42}$, F. Martinez Vidal ${ }^{65}$, D. Martins Tostes ${ }^{2}$, A. Massafferri ${ }^{1}$, R. Matev ${ }^{38}$, Z. Mathe ${ }^{38}$, C. Matteuzzi ${ }^{20}$, A. Mazurov ${ }^{45}$, M. McCann ${ }^{53}$, J. McCarthy ${ }^{45}$, A. $\mathrm{McNab}^{54}$, R. McNulty ${ }^{12}$, B. McSkelly ${ }^{52}$, B. Meadows ${ }^{57}$, F. Meier ${ }^{9}$, M. Meissner ${ }^{11}$, M. Merk ${ }^{41}$, D.A. Milanes ${ }^{62}$, M.-N. Minard ${ }^{4}$, N. Moggi ${ }^{14}$, J. Molina Rodriguez ${ }^{60}$, S. Monteil ${ }^{5}$, M. Morandin ${ }^{22}$, P. Morawski ${ }^{27}$, A. Mordà ${ }^{6}$, M.J. Morello ${ }^{23, t}$, J. Moron ${ }^{27}$, A.-B. Morris ${ }^{50}$, R. Mountain ${ }^{59}$, F. Muheim ${ }^{50}$, K. Müller ${ }^{40}$, M. Mussini ${ }^{14}$, B. Muster ${ }^{39}$, P. Naik $^{46}$, T. Nakada ${ }^{39}$, R. Nandakumar ${ }^{49}$, I. Nasteva ${ }^{2}$, M. Needham ${ }^{50}$, N. Neri ${ }^{21}$, S. Neubert ${ }^{38}$, N. Neufeld ${ }^{38}$, M. Neuner ${ }^{11}$, A.D. Nguyen ${ }^{39}$, T.D. Nguyen ${ }^{39}$, C. Nguyen-Mau ${ }^{39, q}$, M. Nicol ${ }^{7}$,

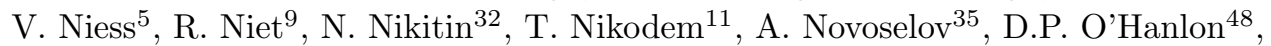

A. Oblakowska-Mucha ${ }^{27}$, V. Obraztsov ${ }^{35}$, S. Ogilvy ${ }^{51}$, O. Okhrimenko ${ }^{44}$, R. Oldeman ${ }^{15, e}$, C.J.G. Onderwater ${ }^{66}$, M. Orlandea ${ }^{29}$, B. Osorio Rodrigues ${ }^{1}$, J.M. Otalora Goicochea ${ }^{2}$, A. Otto ${ }^{38}$, P. Owen ${ }^{53}$, A. Oyanguren ${ }^{65}$, B.K. Pal ${ }^{59}$, A. Palano ${ }^{13, c}$, F. Palombo ${ }^{21, u}$, M. Palutan ${ }^{18}$, J. Panman ${ }^{38}$, A. Papanestis ${ }^{49,38}$, M. Pappagallo ${ }^{51}$, L.L. Pappalardo ${ }^{16, f}$, C. Parkes ${ }^{54}$, C.J. Parkinson ${ }^{9,45}$, G. Passaleva ${ }^{17}$, G.D. Patel ${ }^{52}$, M. Patel ${ }^{53}$, C. Patrignani ${ }^{19, j}$, A. Pearce ${ }^{54}$, A. Pellegrino ${ }^{41}$, G. Penso ${ }^{25, m}$, M. Pepe Altarelli ${ }^{38}$, S. Perazzini ${ }^{14, d}$, P. Perret ${ }^{5}$, L. Pescatore ${ }^{45}$, E. Pesen ${ }^{67}$, K. Petridis ${ }^{53}$, A. Petrolini ${ }^{19, j}$, E. Picatoste Olloqui ${ }^{36}$, B. Pietrzyk ${ }^{4}$, T. Pilaŕ ${ }^{48}$, D. Pinci ${ }^{25}$, A. Pistone ${ }^{19}$, S. Playfer ${ }^{50}$, M. Plo Casasus ${ }^{37}$, F. Polci ${ }^{8}$, A. Poluektov ${ }^{48,34}$, I. Polyakov ${ }^{31}$, E. Polycarpo ${ }^{2}$, A. Popov ${ }^{35}$, D. Popov ${ }^{10}$, B. Popovici ${ }^{29}$, C. Potterat ${ }^{2}$, E. Price ${ }^{46}$, J.D. Price ${ }^{52}$, J. Prisciandaro ${ }^{39}$, A. Pritchard ${ }^{52}$, C. Prouve ${ }^{46}$, V. Pugatch ${ }^{44}$, A. Puig Navarro ${ }^{39}$, G. Punzi ${ }^{23, s}$, W. Qian ${ }^{4}$, B. Rachwal ${ }^{26}$, J.H. Rademacker ${ }^{46}$, B. Rakotomiaramanana ${ }^{39}$, M. Rama ${ }^{23}$, M.S. Rangel ${ }^{2}$, I. Raniuk ${ }^{43}$, N. Rauschmayr ${ }^{38}$, G. Raven ${ }^{42}$, F. Redi ${ }^{53}$, S. Reichert ${ }^{54}$, M.M. Reid ${ }^{48}$, A.C. dos Reis ${ }^{1}$, S. Ricciardi ${ }^{49}$, S. Richards ${ }^{46}$, M. Rihl ${ }^{38}$, K. Rinnert ${ }^{52}$, V. Rives Molina ${ }^{36}$, P. Robbe ${ }^{7}$, A.B. Rodrigues ${ }^{1}$, E. Rodrigues ${ }^{54}$, P. Rodriguez Perez ${ }^{54}$, S. Roiser ${ }^{38}$, V. Romanovsky ${ }^{35}$, A. Romero Vidal ${ }^{37}$, M. Rotondo ${ }^{22}$, J. Rouvinet ${ }^{39}$, T. Ruf ${ }^{38}$, H. Ruiz ${ }^{36}$, P. Ruiz Valls ${ }^{65}$, J.J. Saborido Silva ${ }^{37}$, N. Sagidova ${ }^{30}$, P. Sail ${ }^{51}$, B. Saitta ${ }^{15, e}$, V. Salustino Guimaraes ${ }^{2}$, C. Sanchez Mayordomo ${ }^{65}$, B. Sanmartin Sedes ${ }^{37}$, R. Santacesaria ${ }^{25}$, C. Santamarina Rios ${ }^{37}$, E. Santovetti ${ }^{24, l}$, A. Sarti ${ }^{18, m}$, C. Satriano ${ }^{25, n}$, A. Satta ${ }^{24}$, D.M. Saunders ${ }^{46}$, D. Savrina ${ }^{31,32}$, M. Schiller ${ }^{38}$, H. Schindler ${ }^{38}$, M. Schlupp ${ }^{9}$, M. Schmelling ${ }^{10}$, B. Schmidt ${ }^{38}$, O. Schneider ${ }^{39}$, A. Schopper ${ }^{38}$, M.-H. Schune ${ }^{7}$, R. Schwemmer ${ }^{38}$, B. Sciascia ${ }^{18}$, A. Sciubba ${ }^{25, m}$, A. Semennikov ${ }^{31}$, I. Sepp ${ }^{53}$, N. Serra ${ }^{40}$, J. Serrano ${ }^{6}$, L. Sestini2 ${ }^{22}$, P. Seyfert ${ }^{11}$, M. Shapkin ${ }^{35}$, I. Shapoval ${ }^{16,43, f}$, Y. Shcheglov ${ }^{30}$, T. Shears ${ }^{52}$, L. Shekhtman ${ }^{34}$, V. Shevchenko ${ }^{64}$, 
A. Shires ${ }^{9}$, R. Silva Coutinho ${ }^{48}$, G. Simi ${ }^{22}$, M. Sirendi ${ }^{47}$, N. Skidmore ${ }^{46}$, I. Skillicorn ${ }^{51}$, T. Skwarnicki ${ }^{59}$, N.A. Smith ${ }^{52}$, E. Smith ${ }^{55,49}$, E. Smith ${ }^{53}$, J. Smith ${ }^{47}$, M. Smith ${ }^{54}$, H. Snoek ${ }^{41}$, M.D. Sokoloff ${ }^{57}$, F.J.P. Soler ${ }^{51}$, F. Soomro ${ }^{39}$, D. Souza ${ }^{46}$, B. Souza De Paula ${ }^{2}$, B. Spaan ${ }^{9}$,

P. Spradlin ${ }^{51}$, S. Sridharan ${ }^{38}$, F. Stagni ${ }^{38}$, M. Stahl ${ }^{11}$, S. Stahl ${ }^{11}$, O. Steinkamp ${ }^{40}$, O. Stenyakin ${ }^{35}$, F Sterpka ${ }^{59}$, S. Stevenson ${ }^{55}$, S. Stoica ${ }^{29}$, S. Stone ${ }^{59}$, B. Storaci ${ }^{40}$, S. Stracka ${ }^{23, t}$, M. Straticiuc ${ }^{29}$, U. Straumann ${ }^{40}$, R. Stroili2 ${ }^{22}$, L. Sun ${ }^{57}$, W. Sutcliffe ${ }^{53}$, K. Swientek ${ }^{27}$, S. Swientek ${ }^{9}$, V. Syropoulos ${ }^{42}$, M. Szczekowski ${ }^{28}$, P. Szczypka ${ }^{39,38}$, T. Szumlak ${ }^{27}$, S. T'Jampens ${ }^{4}$, M. Teklishyn ${ }^{7}$, G. Tellarini ${ }^{16, f}$, F. Teubert ${ }^{38}$, C. Thomas ${ }^{55}$, E. Thomas ${ }^{38}$, J. van Tilburg ${ }^{41}$,

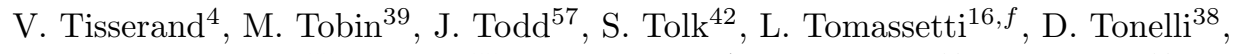
S. Topp-Joergensen ${ }^{55}$, N. Torr ${ }^{55}$, E. Tournefier ${ }^{4}$, S. Tourneur ${ }^{39}$, M.T. $\operatorname{Tran}^{39}$, M. $\operatorname{Tresch}^{40}$, A. Trisovic ${ }^{38}$, A. Tsaregorodtsev 6 , P. Tsopelas ${ }^{41}$, N. Tuning ${ }^{41}$, M. Ubeda Garcia ${ }^{38}$, A. Ukleja ${ }^{28}$, A. Ustyuzhanin ${ }^{64}$, U. Uwer ${ }^{11}$, C. Vacca ${ }^{15}$, V. Vagnoni ${ }^{14}$, G. Valenti ${ }^{14}$, A. Vallier ${ }^{7}$, R. Vazquez Gomez ${ }^{18}$, P. Vazquez Regueiro ${ }^{37}$, C. Vázquez Sierra ${ }^{37}$, S. Vecchi ${ }^{16}$, J.J. Velthuis ${ }^{46}$, M. Veltri ${ }^{17, h}$, G. Veneziano ${ }^{39}$, M. Vesterinen ${ }^{11}$, JVVB Viana Barbosa $^{38}$, B. Viaud ${ }^{7}$, D. Vieira ${ }^{2}$, M. Vieites Diaz ${ }^{37}$, X. Vilasis-Cardona ${ }^{36, p}$, A. Vollhardt ${ }^{40}$, D. Volyanskyy ${ }^{10}$, D. Voong ${ }^{46}$, A. Vorobyev ${ }^{30}$, V. Vorobyev ${ }^{34}$, C. Voß ${ }^{63}$, J.A. de Vries $^{41}$, R. Waldi ${ }^{63}$, C. Wallace ${ }^{48}$, R. Wallace ${ }^{12}$, J. Walsh ${ }^{23}$, S. Wandernoth ${ }^{11}$, J. Wang ${ }^{59}$, D.R. Ward ${ }^{47}$, N.K. Watson ${ }^{45}$, D. Websdale ${ }^{53}$, M. Whitehead ${ }^{48}$, D. Wiedner ${ }^{11}$, G. Wilkinson ${ }^{55,38}$, M. Wilkinson ${ }^{59}$, M.P. Williams ${ }^{45}$, M. Williams ${ }^{56}$, H.W. Wilschut ${ }^{66}$, F.F. Wilson ${ }^{49}$, J. Wimberley ${ }^{58}$, J. Wishahi ${ }^{9}$, W. Wislicki ${ }^{28}$, M. Witek ${ }^{26}$, G. Wormser ${ }^{7}$, S.A. Wotton ${ }^{47}$, S. Wright ${ }^{47}$, K. Wyllie ${ }^{38}, Y$. Xie ${ }^{61}$, Z. Xing ${ }^{59}$, Z. Xu ${ }^{39}$, Z. Yang ${ }^{3}$, X. Yuan ${ }^{3}$, O. Yushchenko ${ }^{35}$, M. Zangoli ${ }^{14}$, M. Zavertyaev ${ }^{10, b}$, L. Zhang ${ }^{3}$, W.C. Zhang ${ }^{12}$, Y. Zhang ${ }^{3}$, A. Zhelezov ${ }^{11}$, A. Zhokhov ${ }^{31}$ and L. Zhong ${ }^{3}$.

Centro Brasileiro de Pesquisas Físicas (CBPF), Rio de Janeiro, Brazil

2 Universidade Federal do Rio de Janeiro (UFRJ), Rio de Janeiro, Brazil

3 Center for High Energy Physics, Tsinghua University, Beijing, China

4 LAPP, Université de Savoie, CNRS/IN2P3, Annecy-Le-Vieux, France

5 Clermont Université, Université Blaise Pascal, CNRS/IN2P3, LPC, Clermont-Ferrand, France

6 CPPM, Aix-Marseille Université, CNRS/IN2P3, Marseille, France

7 LAL, Université Paris-Sud, CNRS/IN2P3, Orsay, France

8 LPNHE, Université Pierre et Marie Curie, Université Paris Diderot, CNRS/IN2P3, Paris, France

9 Fakultät Physik, Technische Universität Dortmund, Dortmund, Germany

10 Max-Planck-Institut für Kernphysik (MPIK), Heidelberg, Germany

11 Physikalisches Institut, Ruprecht-Karls-Universität Heidelberg, Heidelberg, Germany

12 School of Physics, University College Dublin, Dublin, Ireland

13 Sezione INFN di Bari, Bari, Italy

14 Sezione INFN di Bologna, Bologna, Italy

15 Sezione INFN di Cagliari, Cagliari, Italy

16 Sezione INFN di Ferrara, Ferrara, Italy

17 Sezione INFN di Firenze, Firenze, Italy

18 Laboratori Nazionali dell'INFN di Frascati, Frascati, Italy

19 Sezione INFN di Genova, Genova, Italy

20 Sezione INFN di Milano Bicocca, Milano, Italy

21 Sezione INFN di Milano, Milano, Italy

22 Sezione INFN di Padova, Padova, Italy

23 Sezione INFN di Pisa, Pisa, Italy

24 Sezione INFN di Roma Tor Vergata, Roma, Italy

25 Sezione INFN di Roma La Sapienza, Roma, Italy

26 Henryk Niewodniczanski Institute of Nuclear Physics Polish Academy of Sciences, Kraków, Poland

27 AGH - University of Science and Technology, Faculty of Physics and Applied Computer Science, Kraków, Poland 
28 National Center for Nuclear Research (NCBJ), Warsaw, Poland

29 Horia Hulubei National Institute of Physics and Nuclear Engineering, Bucharest-Magurele, Romania

30 Petersburg Nuclear Physics Institute (PNPI), Gatchina, Russia

31 Institute of Theoretical and Experimental Physics (ITEP), Moscow, Russia

32 Institute of Nuclear Physics, Moscow State University (SINP MSU), Moscow, Russia

33 Institute for Nuclear Research of the Russian Academy of Sciences (INR RAN), Moscow, Russia

34 Budker Institute of Nuclear Physics (SB RAS) and Novosibirsk State University, Novosibirsk, Russia

35 Institute for High Energy Physics (IHEP), Protvino, Russia

36 Universitat de Barcelona, Barcelona, Spain

37 Universidad de Santiago de Compostela, Santiago de Compostela, Spain

38 European Organization for Nuclear Research (CERN), Geneva, Switzerland

39 Ecole Polytechnique Fédérale de Lausanne (EPFL), Lausanne, Switzerland

40 Physik-Institut, Universität Zürich, Zürich, Switzerland

41 Nikhef National Institute for Subatomic Physics, Amsterdam, The Netherlands

42 Nikhef National Institute for Subatomic Physics and VU University Amsterdam, Amsterdam, The Netherlands

43 NSC Kharkiv Institute of Physics and Technology (NSC KIPT), Kharkiv, Ukraine

44 Institute for Nuclear Research of the National Academy of Sciences (KINR), Kyiv, Ukraine

45 University of Birmingham, Birmingham, United Kingdom

46 H.H. Wills Physics Laboratory, University of Bristol, Bristol, United Kingdom

47 Cavendish Laboratory, University of Cambridge, Cambridge, United Kingdom

48 Department of Physics, University of Warwick, Coventry, United Kingdom

49 STFC Rutherford Appleton Laboratory, Didcot, United Kingdom

50 School of Physics and Astronomy, University of Edinburgh, Edinburgh, United Kingdom

51 School of Physics and Astronomy, University of Glasgow, Glasgow, United Kingdom

52 Oliver Lodge Laboratory, University of Liverpool, Liverpool, United Kingdom

53 Imperial College London, London, United Kingdom

54 School of Physics and Astronomy, University of Manchester, Manchester, United Kingdom

55 Department of Physics, University of Oxford, Oxford, United Kingdom

56 Massachusetts Institute of Technology, Cambridge, MA, United States

57 University of Cincinnati, Cincinnati, OH, United States

58 University of Maryland, College Park, MD, United States

59 Syracuse University, Syracuse, NY, United States

60 Pontifícia Universidade Católica do Rio de Janeiro (PUC-Rio), Rio de Janeiro, Brazil, associated to ${ }^{2}$

61 Institute of Particle Physics, Central China Normal University, Wuhan, Hubei, China, associated to ${ }^{3}$

62 Departamento de Fisica, Universidad Nacional de Colombia, Bogota, Colombia, associated to ${ }^{8}$

63 Institut für Physik, Universität Rostock, Rostock, Germany, associated to ${ }^{11}$

64 National Research Centre Kurchatov Institute, Moscow, Russia, associated to ${ }^{31}$

65 Instituto de Fisica Corpuscular (IFIC), Universitat de Valencia-CSIC, Valencia, Spain, associated to ${ }^{36}$

66 Van Swinderen Institute, University of Groningen, Groningen, The Netherlands, associated to ${ }^{41}$

67 Celal Bayar University, Manisa, Turkey, associated to ${ }^{38}$

a Universidade Federal do Triângulo Mineiro (UFTM), Uberaba-MG, Brazil

${ }^{b}$ P.N. Lebedev Physical Institute, Russian Academy of Science (LPI RAS), Moscow, Russia

c Università di Bari, Bari, Italy

d Università di Bologna, Bologna, Italy

e Università di Cagliari, Cagliari, Italy 
$f$ Università di Ferrara, Ferrara, Italy

$g$ Università di Firenze, Firenze, Italy

$h$ Università di Urbino, Urbino, Italy

i Università di Modena e Reggio Emilia, Modena, Italy

j Università di Genova, Genova, Italy

${ }^{k}$ Università di Milano Bicocca, Milano, Italy

$l$ Università di Roma Tor Vergata, Roma, Italy

$m$ Università di Roma La Sapienza, Roma, Italy

$n$ Università della Basilicata, Potenza, Italy

o AGH - University of Science and Technology, Faculty of Computer Science, Electronics and Telecommunications, Kraków, Poland

$p$ LIFAELS, La Salle, Universitat Ramon Llull, Barcelona, Spain

$q$ Hanoi University of Science, Hanoi, Viet Nam

$r$ Università di Padova, Padova, Italy

$s$ Università di Pisa, Pisa, Italy

${ }^{t}$ Scuola Normale Superiore, Pisa, Italy

u Università degli Studi di Milano, Milano, Italy

$v$ Politecnico di Milano, Milano, Italy 